\title{
TRITIUM IN GROUND WATER IN MISSISSIPPI,
}

1989-90

By Larry J. Slack and William T. Oakley

U.S. GEOLOGICAL SURVEY

Open-File Report 91-177

Prepared in cooperation with the

MISSISSIPPI DEPARTMENT OF ENVIRONMENTAL QUALITY, OFFICE OF POLLUTION CONTROL

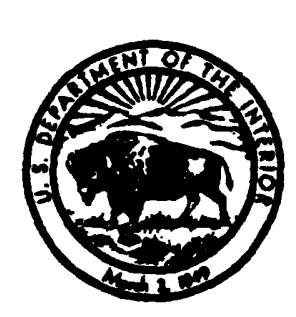

Jackson, Mississippi

1991 


\section{U.S. DEPARTMENT OF THE INTERIOR \\ MANUEL LUJAN, JR., Secretary}

U.S. GEOLOGICAL SURVEY

Dallas L. Peck, Director

For additional information write to:

District Chief

U.S. Geological Survey

Suite 710, Federal Building $100 \mathrm{~W}$. Capitol Street

Jackson, Mississippi 39269
Copies of this report can be purchased from:

U.S. Geological Survey Books and Open-File Reports

Building 810

Box 25425, Federal Center

Denver, Colorado 80225 


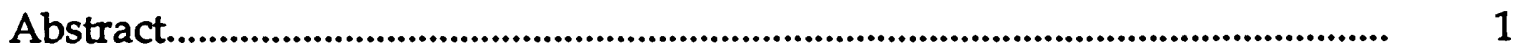

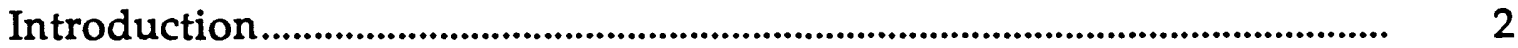

Purpose and scope......................................................................................... 2

Use of tritium as a tracer .............................................................................

Site selection criteria and site information..................................................... 3

Methods of sampling and analysis............................................................................ 3

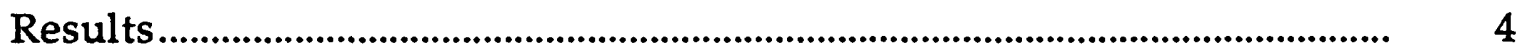

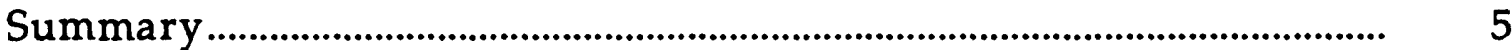

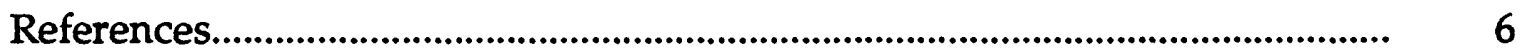

\section{ILLUSTRATIONS}

Figure 1. Map showing location of ground-water sites with tritium analyses.

2. Map showing tritium concentrations in ground water

\section{TABLES}

Table 1. Geographic units and principal aquifers in Mississippi.............. 9

2. Summary of site information............................................................ 10

3. Values for tritium and selected water-quality properties and constituents for ground water.

4. Median values of depth, specific conductance, $\mathrm{pH}$, and alkalinity for pre- and post-1953 ground water (based on 1989-90 tritium data).

\section{CONVERSION FACTORS}

$\begin{array}{lcl}\text { Multiply } & \text { By } & \text { To obtain } \\ \text { foot } & 25.4 & \text { millimeter } \\ \text { gallon per minute } & 0.06308 & \text { liter per second }\end{array}$




\title{
TRITIUM IN GROUND WATER IN MISSISSIPPI,
}

\author{
1989-90
}

\author{
by Larry J. Slack and William T. Oakley
}

\begin{abstract}
In April 1989 and in April and May 1990, the U.S. Geological Survey collected water samples from 43 shallow wells (less than 500 feet deep) for tritium analyses; the wells are completed in 13 of the 14 principal aquifers in Mississippi. Tritium concentrations ranged from less than 0.3 to 42 picocuries per liter. Tritium concentrations in water from about 40 percent of the wells were greater than 1 picocurie per liter, indicating modern (post-1953) water. The median tritium concentration for wells with modern water was 21 picocuries per liter; the mean, 23 picocuries per liter.

The median depth, specific conductance, $p H$, and alkalinity values were substantially smaller for the wells with modern water than for wells with older (pre-1953) water. Wells with modern water had a median depth of 187 feet; specific conductance, 66 microsiemens per centimeter at 25 degrees Celsius; $p H, 5.9$; and alkalinity as calcium carbonate, 10 milligrams per liter. Wells with pre-1953 water had a median depth of 277 feet; specific conductance, 339 microsiemens; $p H$, 7.6; and alkalinity, 158 milligrams per liter.
\end{abstract}




\section{INTRODUCTION}

As part of the Federal-State Cooperative Program, the U.S. Geological Survey (USGS) collects, on a systematic basis, data needed for the continuing determination and evaluation of the quantity, quality, and use of water resources in the United States, and appraises the availability of ground and surface water through analytical and interpretive investigations. The resulting information forms the foundation for many of the Nation's water-resources management and planning activities and allows for the detection of emerging water problems.

The Mississippi Department of Environmental Quality, Office of Pollution Control (OPC), is the principal agency responsible for the management and protection of ground water in Mississippi. The OPC administers programs and policies to secure, protect, and preserve the right of citizens to unpolluted waters. Together with the Mississippi Department of Agriculture and Commerce, the OPC is developing a comprehensive program to protect aquifers from surface and shallowsource contamination. The USGS, in cooperation with these agencies, is conducting an investigation to describe the relative susceptibility of major aquifers in Mississippi to surface and shallow-source contamination.

\section{Purpose and Scope}

One of the objectives of the OPC's ground-water protection programs is to investigate the relative age of ground water in the principal aquifers in Mississippi that are associated with the Well Head Protection Program. In 1989 and 1990, in cooperation with the OPC, the USGS used radioisotope (tritium) dating techniques to assess the relative age of shallow ground water for selected aquifers in the State. This report summarizes the site information, methods of sampling and analysis, and results of the tritium analyses and other selected water-quality data collected as part of that study. 


\section{Use of Tritium as a Tracer}

Tritium has been used extensively as a hydrologic tracer since the early 1950 's and can be used to indicate the relative age of water (pre- or post1953). Tritium is particularly useful in ground-water studies because it is relatively unaffected by reactions other than radioactive decay. Tritium is a radioactive isotope of hydrogen with an atomic weight of 3 and a halflife of $\mathbf{1 2 . 4 3}$ years. Tritium is produced naturally and occurs in a small but nearly constant concentration in the atmosphere (Lal and Peters, 1967). Prior to the initiation of atmospheric testing of large thermonuclear weapons in 1953, the natural tritium content of rainwater was about 1 to 5 Tritium Units or 1 to 5 tritium atoms per $10^{18}$ normal hydrogen atoms (Thatcher, 1962, p. 48). In the 1950's and 1960's, tritium concentrations in precipitation in the northern hemisphere increased substantially and resulted in values of about 50 Tritium Units for surface ocean water and 100 's or 1000's of Tritium Units for some continental water (Michel, 1989, p. 2).

\section{SITE SELECTION CRITERIA AND SITE INFORMATION}

In April 1989 and in April and May 1990, the USGS collected water samples from 43 shallow wells (less than 500 feet deep) (fig. 1) for tritium analyses; the wells were completed in 17 geologic units in 13 of the 14 principal aquifers in Mississippi (tables 1 and 2). The availability of complete and accurate well-construction information was an essential selection criterion for each site; consequently, first preference was given to public-supply wells for which driller's logs and geophysical logs are available. Other selection criteria included wells that were in use, had turbine or submersible pumps, and had a yield of at least 25 gallons per minute.

\section{METHODS OF SAMPLING AND ANALYSIS}

To assure that samples were representative of water from the waterbearing unit, the wells were pumped long enough prior to sampling to 
evacuate at least twice the volume of water standing in the casing. Most of the wells sampled were in daily operation, and some were in continuous operation.

Water samples for tritium analysis were collected in a narrow-mouth flint glass bottle with a polyseal cap. The bottle was completely filled, with care taken not to entrain air. The bottle was capped, and the cap was taped to prevent it from loosening during transit.

The samples were shipped to the USGS Water Resources Division National Water Quality Laboratory in Denver, Colorado, where they were recorded, repackaged, and forwarded to the University of Miami Tritium Laboratory. At the Tritium Laboratory, the samples were analyzed by an electrolytic enrichment with gas counting method developed by Ostlund and Werner (1961). It is the most sensitive method of tritium analysis available through the National Water Quality Laboratory. The lower detection limit is 0.3 picocurie per liter.

\section{RESULTS}

Tritium concentrations for the 43 wells (table 3) are in picocuries per liter; for the convenience of the reader, Tritium Units (which were obtained by dividing picocuries per liter by a conversion factor of 3.2) are also shown. Laboratory values for specific conductance, $\mathrm{pH}$, and alkalinity for water from the 43 wells are also included in table 3.

Tritium concentrations less than about 1 picocurie per liter are considered to represent water with natural or background levels of tritium for ground water in Mississippi (R.L. Michel, USGS, oral commun., September 13, 1989). Values greater than 1 picocurie per liter are considered to represent post-1953 water, which is commonly referred to as "modern water" or "bomb tritium water." The limited amount of tritium data obtained during this study precludes drawing conclusions about the typical concentrations for any given aquifer.

Tritium concentrations ranged from less than 0.3 (the lower detection limit) to 42 picocuries per liter (table 3 ; fig. 2). Tritium concentrations in water from 24 of the wells were less than the lower detection limit. In 17 of the 43 wells (about 40 percent), tritium concentrations were greater than 1 picocurie per liter, indicating modern (post-1953) water. The median 
tritium concentration for wells with modern water was 21 picocuries per liter; the mean, 23 picocuries per liter. The largest concentrations of tritium detected in ground water during this study are approximately equal to that in present-day (1991) precipitation in the area, or about 30 to 40 picocuries per liter (R.L. Michel, USGS, written commun., January 26, 1991).

The median depth, specific conductance, $\mathrm{pH}$, and alkalinity values were substantially smaller for the wells with modern water than for wells with older (pre-1953) water (table 4). Wells with modern water had depths ranging from 96 to 360 feet, and a median depth of 187 feet. Water from these modern-water wells had specific conductance values ranging from 20 to 558 microsiemens and a median of 66 microsiemens. Water from these wells had $\mathrm{pH}$ values ranging from 5.3 to 7.5, and a median $\mathrm{pH}$ of 5.9. Water from these wells had alkalinity values (as calcium carbonate) ranging from 2 to 288 milligrams per liter, and a median of 10 milligrams per liter.

Wells with pre-1953 water had depths ranging from 138 to 483 feet, and a median depth of 277 feet. Water from these wells had specific conductance values ranging from 30 to 938 microsiemens, and a median of 339 microsiemens. Water from these wells had $\mathrm{pH}$ values ranging from 6.2 to 8.6, and a median $\mathrm{pH}$ of 7.6. Water from these wells had alkalinity values (as calcium carbonate) ranging from 7 to 299 milligrams per liter, and a median of 158 milligrams per liter.

\section{SUMMARY}

In April 1989 and in April and May 1990, the U.S. Geological Survey collected water samples from 43 shallow wells (less than 500 feet deep) for tritium analyses; the wells are completed in 13 of the 14 principal aquifers in Mississippi. Tritium concentrations ranged from less than 0.3 to 42 picocuries per liter. Tritium concentrations in water from about 40 percent of the wells were greater than 1 picocurie per liter, indicating modern (post-1953) water. The median depth, specific conductance, $\mathrm{pH}$, and alkalinity values were substantially smaller for the wells with modern water than for wells with older (pre-1953) water. 


\section{REFERENCES}

Lal, D., and Peters, B., 1967, Cosmic ray produced radioactivity on the earth, in Flugge, S., ed., Encyclopedia of Physics, v. 46/2, p. 551-612.

Michel, R.L., 1989, Tritium deposition in the continental United States, 1953-83: U.S. Geological Survey Water-Resources Investigations Report 89-4072, 51 p.

Ostlund, H.G., and Werner, E., 1961, The electrolytic enrichment of tritium and deuterium for natural tritium measurements: Tritium in the Physical and Biological Sciences, Proceedings, vol. 1, p. 95-105.

Slack, L.J., and Darden, Daphne, 1991, Summary of aquifer tests in Mississippi, June 1942 through May 1988: U.S. Geological Survey Open-File Report 90-4155, 40 p.

Thatcher, L.L., 1962, The distribution of tritium fallout in precipitation over North America: International Association of Science Hydrology Bulletin, vol. 7, no. 2, p. 48.

Wasson, B.E., 1986, Sources for water supplies in Mississippi: Mississippi Research and Development Center, 113 p. 


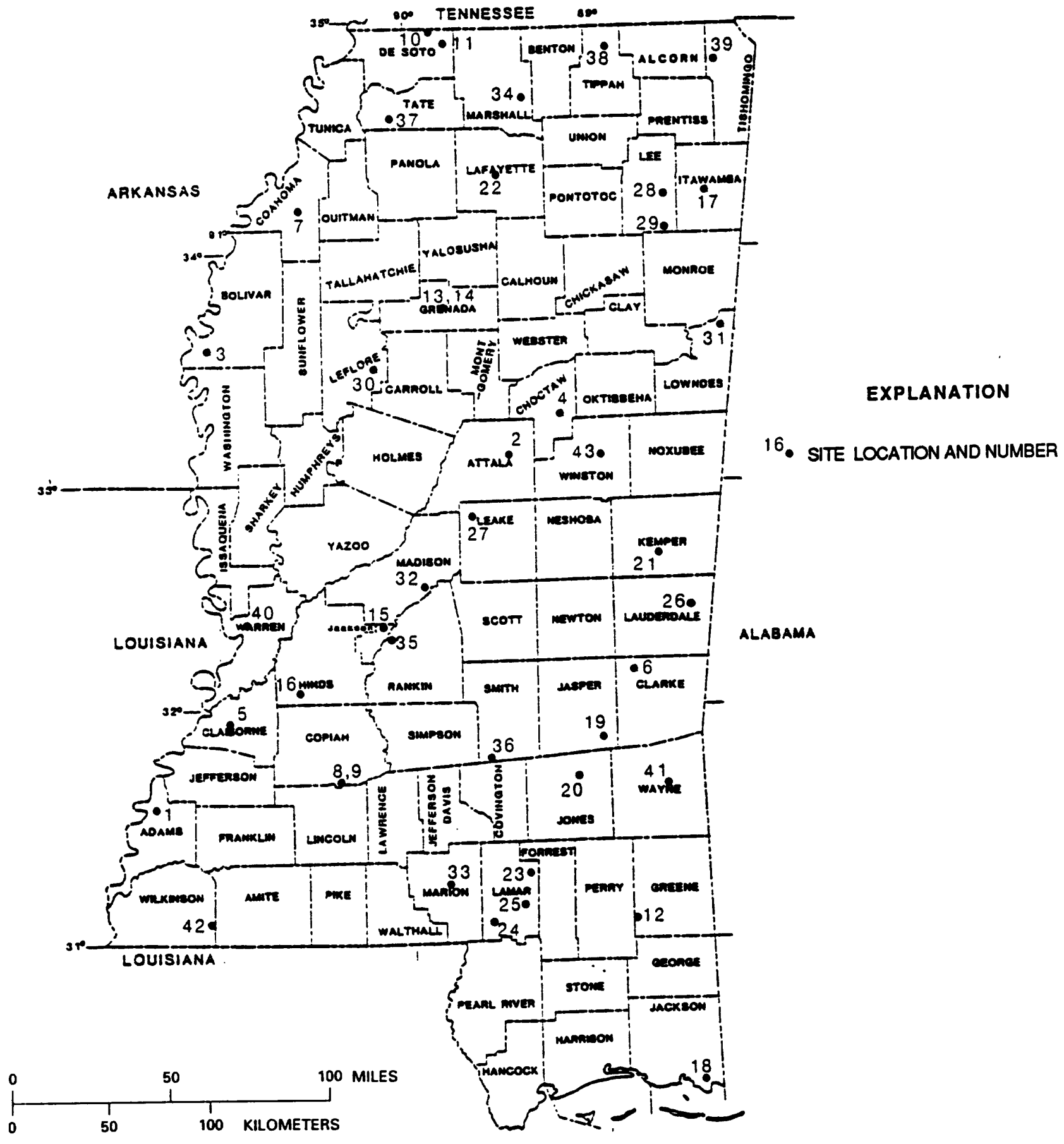

Figure 1.-Location of ground-water sites with tritium analyses. 


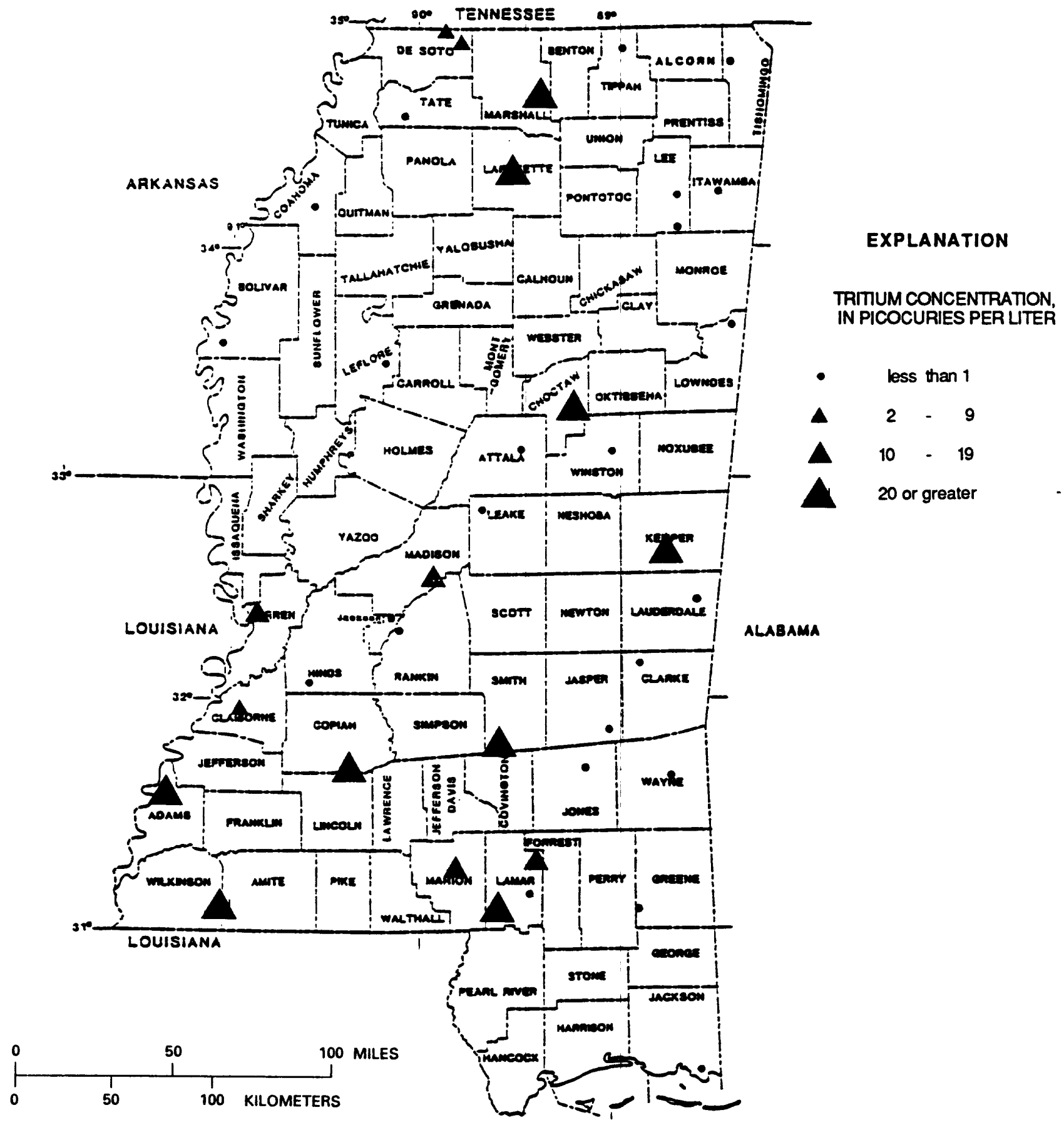

Figure 2.--Tritium concentrations in ground water. 
Table 1. Geologic units and principal aquifers in Mississippi

[Modified from Slack and Darden, 1991; only geologic unit codes for units

tabulated in table 2 are listed]

\begin{tabular}{|c|c|c|c|c|c|c|}
\hline Erathem & System & Series & Group & $\begin{array}{l}\text { Geologic } \\
\text { unit } \\
\text { code }\end{array}$ & Geologic unit & $\begin{array}{l}\text { Princlpal equifer } \\
\text { or aquifer system }\end{array}$ \\
\hline \multirow{14}{*}{ Cenozoic } & \multirow[t]{2}{*}{ Quatemary } & $\begin{array}{l}\text { Holocene } \\
\text { and } \\
\text { Pleistocene }\end{array}$ & & MRVA & $\begin{array}{l}\text { Quatemary alluvium } \\
\text { Missiesippi River } \\
\text { valley alluvium }\end{array}$ & $\begin{array}{l}\text { Miscissippi River } \\
\text { alluvial aquifer }\end{array}$ \\
\hline & & Pleistocene & & & $\begin{array}{l}\text { Loess } \\
\text { Temrace deposits }\end{array}$ & \\
\hline & \multirow{12}{*}{ Tertiary } & Pliocene & & $\begin{array}{l}\text { CRNL } \\
\text { GRMF }\end{array}$ & $\begin{array}{l}\text { Citronelle Formation } \\
\text { Graham Ferry Formation }\end{array}$ & Citronelle aquifers \\
\hline & & Miocene & & $\begin{array}{l}\text { CTHLL } \\
\text { MOCN }\end{array}$ & $\begin{array}{l}\text { Pascagoula Formation } \\
\text { Hattiesburg Formation } \\
\text { Catahoula Sandstone, upper part } \\
\text { Catahoula Sandstone, lower part } \\
\text { Deposits of Miocene age }\end{array}$ & Miocene aquifer system \\
\hline & & Oligocene & $\begin{array}{l}\text { Vicksburg } \\
\text { Group }\end{array}$ & WRBR & $\begin{array}{l}\text { Bucatunna Formation } \\
\text { Waynesboro sand lentil } \\
\text { Byram Formation } \\
\text { Glendon Limestone } \\
\text { Marianna Formation } \\
\text { Mint Spring Formation }\end{array}$ & $\begin{array}{l}\text { Oligocene aquifer } \\
\text { system }\end{array}$ \\
\hline & & & & & Forest Hill Formation & \\
\hline & & \multirow[t]{6}{*}{ Eocene } & $\begin{array}{l}\text { Jackson } \\
\text { Group }\end{array}$ & & $\begin{array}{l}\text { Yazoo Clay } \\
\text { Moodys Branch Formation }\end{array}$ & \\
\hline & & & $\begin{array}{l}\text { Claiborne } \\
\text { Group }\end{array}$ & CCKF & $\begin{array}{l}\text { Cockfield Formation } \\
\text { Cook Mountuin Formation }\end{array}$ & $\begin{array}{l}\text { Cockfield aquifer } \\
\text { Soart aquifer }\end{array}$ \\
\hline & & & & & Zilpha Clay & 30 . \\
\hline & & & & THLT & $\begin{array}{l}\text { Winona Sand } \\
\text { Tallahatu Formation } \\
\text { Neshobe Sand Member } \\
\text { Basic City Shale Member }\end{array}$ & $\begin{array}{l}\text { Wincona-Tallahata } \\
\text { aquifer }\end{array}$ \\
\hline & & & & MuwX & $\begin{array}{l}\text { Meridian Sand Member } \\
\text { Meridian Sand Member } \\
\text { and Wilcox Group, upper par }\end{array}$ & $\begin{array}{l}\text { Meridian-upper Wilcox } \\
\text { aquifer }\end{array}$ \\
\hline & & & & & $\begin{array}{l}\text { Wilcox Group, upper part } \\
\text { Hatchetigbee Formation }\end{array}$ & \\
\hline & & \multirow[t]{2}{*}{ Paleocene } & $\begin{array}{l}\text { Wilcox } \\
\text { Group }\end{array}$ & $\begin{array}{l}\text { WLCXM } \\
\text { WLCXL }\end{array}$ & $\begin{array}{l}\text { Tuscahoma Formation } \\
\text { Wilcox Group, middle part } \\
\text { Nanufalia Formation } \\
\text { Feam Springs Member } \\
\text { Wilcox Group, lower par }\end{array}$ & Lower Wilcox aquifer \\
\hline & & & $\begin{array}{l}\text { Midway } \\
\text { Group }\end{array}$ & & $\begin{array}{l}\text { Naheole Formation } \\
\text { Porters Creek Clay } \\
\text { Mathews Landing Marl Member } \\
\text { Clayton Formation }\end{array}$ & \\
\hline \multirow[t]{5}{*}{ Mesozoic } & \multirow[t]{5}{*}{ Cretaceous } & \multirow{4}{*}{$\begin{array}{l}\text { Upper } \\
\text { Cretaceous }\end{array}$} & Selma & RPLY & $\begin{array}{l}\text { Praivie Bluff Chalk } \\
\text { and Owl Creek Formation } \\
\text { Ripley Formation }\end{array}$ & Ripley aquifer \\
\hline & & & Group & & $\begin{array}{l}\text { Demopolis Chalk } \\
\text { Coffee Sand } \\
\text { Mooreville Chalk } \\
\text { Arcola Limestone Member }\end{array}$ & Coffee Sand aquifer \\
\hline & & & $\begin{array}{l}\text { Tuscaloosa } \\
\text { Group }\end{array}$ & $\begin{array}{l}\text { EUTW } \\
\text { MCSN }\end{array}$ & $\begin{array}{l}\text { Eutaw Fomation } \\
\text { Tombigbee Sand Member } \\
\text { Eutaw Formation, lower part } \\
\text { MeShan Fomation }\end{array}$ & $\begin{array}{l}\text { Eutaw-McShan } \\
\text { aquifer }\end{array}$ \\
\hline & & & & GORD & $\begin{array}{l}\text { Gordo Formation } \\
\text { Coker Formation } \\
\text { Massive sand }\end{array}$ & $\begin{array}{l}\text { Tuscaloosa aquifer } \\
\text { system }\end{array}$ \\
\hline & & \begin{tabular}{|l|} 
Lower \\
Cretuceous \\
\end{tabular} & & & Undifferentiated & \\
\hline Palcoeoic & & & & PLZC & $\begin{array}{l}\text { Undifferentiated } \\
\text { Paloocoic Enthem }\end{array}$ & $\begin{array}{l}\text { Paleoroic aquifex } \\
\text { system }\end{array}$ \\
\hline
\end{tabular}


Table 2. Summary of site information

[Util, Utilities; W A, Water Association]

\begin{tabular}{|c|c|c|c|c|c|c|c|c|}
\hline $\begin{array}{c}\text { Site } \\
\text { number }\end{array}$ & County & $\begin{array}{l}\text { Local } \\
\text { well } \\
\text { number }\end{array}$ & $\begin{array}{l}\text { Owner or } \\
\text { operator }\end{array}$ & Latitude & Longinde & $\begin{array}{l}\text { Depth } \\
\text { of } \\
\text { well } \\
\text { (feex) }\end{array}$ & $\begin{array}{l}\text { Depth } \\
\text { to lop } \\
\text { of open } \\
\text { interval } \\
\text { (foet) }\end{array}$ & $\begin{array}{l}\text { Geciogic } \\
\text { unit code } \\
\text { (see table 1) }\end{array}$ \\
\hline $\begin{array}{l}1 \\
2 \\
3 \\
4\end{array}$ & $\begin{array}{l}\text { ADAMS } \\
\text { ATTALA } \\
\text { BOLIVAR } \\
\text { CHOCTAW }\end{array}$ & $\begin{array}{l}\text { DOA5 } \\
\text { HO23 } \\
\text { RO74 } \\
\text { HOSO }\end{array}$ & $\begin{array}{l}\text { BROADMOORE UTIL } \\
\text { ETHEL } \\
\text { SCOTT } \\
\text { ACKERMAN }\end{array}$ & $\begin{array}{l}31^{\circ} 355^{\circ} 38^{\prime \prime} \\
33^{\circ} 0730^{\circ} \\
33^{\circ} 35^{\circ} 24^{\prime \prime} \\
33^{\circ} 18^{\circ} 46^{\prime \prime}\end{array}$ & $\begin{array}{l}91^{\circ} 21^{\prime} 32^{\prime \prime} \\
89^{\circ} 28^{\prime} 01^{\prime \prime} \\
91^{\circ} 04^{\prime} 37^{\prime \prime} \\
89^{\circ} 10^{\circ} 34^{\prime \prime}\end{array}$ & $\begin{array}{l}150 \\
210 \\
483 \\
101\end{array}$ & $\begin{array}{l}135 \\
170 \\
431 \\
64\end{array}$ & $\begin{array}{l}\text { MOCN } \\
\text { TLLT } \\
\text { CCKF } \\
\text { WLCXM }\end{array}$ \\
\hline $\begin{array}{l}5 \\
6 \\
7 \\
8\end{array}$ & $\begin{array}{l}\text { CLAIBORNE } \\
\text { CLARKE } \\
\text { COAHOMA } \\
\text { COPLA }\end{array}$ & $\begin{array}{l}2079 \\
\text { A002 } \\
3009 \\
\text { V019 }\end{array}$ & $\begin{array}{l}\text { PORT GIBSON } \\
\text { ENTERPRISE } \\
\text { CLARKSDALE } \\
\text { WESSON }\end{array}$ & $\begin{array}{l}31^{\circ} 5719^{*} \\
32^{\circ} 10^{\prime} 19^{\prime \prime} \\
34^{*} 1212^{\prime \prime} \\
31^{\circ} 4229^{\prime \prime}\end{array}$ & $\begin{array}{l}90^{\circ} 58^{\circ} 52^{\prime \prime} \\
88^{\circ} 49^{\prime} 09^{\prime \prime} \\
9^{\circ} 34^{\prime} 15^{\prime \prime} \\
90^{\circ} 23^{\prime} 14^{\prime \prime}\end{array}$ & $\begin{array}{l}170 \\
250 \\
357 \\
332\end{array}$ & $\begin{array}{l}110 \\
200 \\
307 \\
292\end{array}$ & $\begin{array}{l}\text { CIHIL } \\
\text { MUWX } \\
\text { SPRT } \\
\text { CIHIL }\end{array}$ \\
\hline $\begin{array}{r}9 \\
10 \\
11 \\
12\end{array}$ & $\begin{array}{l}\text { COPLAH } \\
\text { DE SOTO } \\
\text { DE SOTO } \\
\text { GREENE }\end{array}$ & $\begin{array}{l}\text { Vo25 } \\
\text { DO22 } \\
\text { HO73 } \\
\text { NO44 }\end{array}$ & $\begin{array}{l}\text { WESSON } \\
\text { MINERAL WELLS W A } \\
\text { FAIRHAVEN WA } \\
\text { MCLAIN }\end{array}$ & $\begin{array}{l}31^{\circ} 4237^{\prime \prime} \\
34^{\dagger} 59^{\circ} 10^{\prime \prime} \\
34^{\circ} 5559^{\prime \prime} \\
31^{\circ} 0505^{\prime \prime}\end{array}$ & $\begin{array}{l}90^{\circ} 23^{\prime} 10^{\prime \prime} \\
89^{\circ} 51^{\prime} 30^{\prime \prime} \\
89^{\circ} 47^{\prime \prime} 35^{\prime \prime} \\
88^{\circ} 48^{\prime} 58^{\prime \prime}\end{array}$ & $\begin{array}{l}360 \\
272 \\
254 \\
180\end{array}$ & $\begin{array}{l}320 \\
227 \\
214 \\
140\end{array}$ & $\begin{array}{l}\text { CTHI } \\
\text { SPRT } \\
\text { SPRT } \\
\text { MOCN }\end{array}$ \\
\hline $\begin{array}{l}13 \\
14 \\
15 \\
16\end{array}$ & $\begin{array}{l}\text { GRENADA } \\
\text { GRENADA } \\
\text { HINDS } \\
\text { HINDS }\end{array}$ & $\begin{array}{l}\text { HO10 } \\
\mathrm{H} 013 \\
\mathrm{H} 178 \\
\mathrm{SO16}\end{array}$ & $\begin{array}{l}\text { GRENADA } \\
\text { GRENADA } \\
\text { BEST WESTERN } \\
\text { UTICA }\end{array}$ & $\begin{array}{l}33^{\circ} 4643^{\prime \prime} \\
33^{\circ} 41^{\prime} 52^{\prime \prime} \\
32^{\circ} 23^{\prime} 18^{\prime \prime} \\
32^{\circ} 05^{\circ} 40^{\prime \prime}\end{array}$ & $\begin{array}{l}89^{\circ} 48^{\prime} 00^{\prime \prime} \\
89^{\circ} 43^{\circ} 35^{\prime \prime} \\
9^{\circ} 08^{\prime} 46^{\prime \prime} \\
9^{\circ} 35^{\prime} 58^{\prime \prime}\end{array}$ & $\begin{array}{l}172 \\
170 \\
390 \\
298\end{array}$ & $\begin{array}{l}132 \\
145 \\
320 \\
258\end{array}$ & $\begin{array}{l}\text { MUwX } \\
\text { MUWX } \\
\text { OCKF } \\
\text { CTHIL }\end{array}$ \\
\hline $\begin{array}{l}17 \\
18 \\
19 \\
20\end{array}$ & $\begin{array}{l}\text { TTAWAMBA } \\
\text { JACKSON } \\
\text { JASPER } \\
\text { JONES }\end{array}$ & $\begin{array}{l}\mathbf{1 0 2 1} \\
\mathbf{Q 4 2 0} \\
\mathbf{0 0 2} \\
\mathrm{C} 167\end{array}$ & $\begin{array}{l}\text { FULTON } \\
\text { PASCACOULA } \\
\text { HEIDELBERG } \\
\text { LAUREL }\end{array}$ & $\begin{array}{l}34^{\circ} 1628^{*} \\
30^{\circ} 2247^{\prime \prime} \\
31^{\circ} 53^{\prime} 35^{\prime \prime} \\
31^{\circ} 4232^{\prime \prime}\end{array}$ & $\begin{array}{l}88^{\circ} 23^{\circ} 33^{\prime \prime} \\
88^{\circ} 3029^{\prime \prime} \\
88^{\circ} 59^{\circ} 06^{\prime \prime} \\
89^{\circ} 06^{\prime} 52^{\prime \prime}\end{array}$ & $\begin{array}{l}274 \\
346 \\
360 \\
322\end{array}$ & $\begin{array}{l}223 \\
266 \\
300 \\
268\end{array}$ & $\begin{array}{l}\text { GORD } \\
\text { GRMF } \\
\text { CCKF } \\
\text { CIHIL }\end{array}$ \\
\hline $\begin{array}{l}21 \\
22 \\
23 \\
24\end{array}$ & $\begin{array}{l}\text { KEMPER } \\
\text { LAFAYETIE } \\
\text { LAMAR } \\
\text { LAMAR }\end{array}$ & $\begin{array}{l}\text { N016 } \\
\text { F023 } \\
\text { E209 } \\
\text { J276 }\end{array}$ & $\begin{array}{l}\text { KIPLNG W A } \\
\text { OXRORD } \\
\text { NLAMAR WA } \\
\text { NLUMBERTON W A }\end{array}$ & $\begin{array}{l}32^{\prime} 4051^{\prime \prime} \\
34^{\circ} 21^{\prime} 55^{\prime \prime} \\
31^{\circ} 1730^{\prime \prime} \\
31^{\circ} 05^{\prime} 14^{\prime \prime}\end{array}$ & $\begin{array}{l}88^{\circ} 40^{\prime} 26^{\prime \prime} \\
89^{\circ} 30^{\prime} 40^{\prime \prime} \\
89^{\circ} 24^{\prime} 10^{\prime \prime} \\
89^{\circ} 35^{\prime} 53^{\prime \prime}\end{array}$ & $\begin{array}{c}178 \\
96 \\
187 \\
202\end{array}$ & $\begin{array}{r}138 \\
71 \\
147 \\
160\end{array}$ & $\begin{array}{l}\text { WLCXI } \\
\text { MUWX } \\
\text { CRNL } \\
\text { CRNL }\end{array}$ \\
\hline $\begin{array}{l}25 \\
26 \\
27 \\
28\end{array}$ & $\begin{array}{l}\text { LAMAR } \\
\text { LAUDERDALE } \\
\text { LEAKE } \\
\text { LEE }\end{array}$ & $\begin{array}{l}\mathrm{L} 093 \\
\mathrm{~J} 125 \\
\text { A006 } \\
\mathrm{H} 093\end{array}$ & $\begin{array}{l}\text { PROGRESS W A } \\
\text { TOOMSUBA W A } \\
\text { THOMASTOWN } \\
\text { MOORE-RICH W A }\end{array}$ & $\begin{array}{l}31^{\prime} 09^{\prime} 30^{\prime \prime} \\
32^{\prime} 28^{\prime} 10^{\prime \prime} \\
32^{\circ} 51^{\prime} 56^{\prime \prime} \\
34^{\prime \prime} 1546^{\prime \prime}\end{array}$ & $\begin{array}{l}89^{\circ} 25^{\prime} 11^{\prime \prime} \\
88^{\circ} 31^{\circ} 38^{\prime \prime} \\
89^{\circ} 40^{\circ} 03^{\prime \prime} \\
88^{\circ} 36^{\prime} 58^{\prime \prime}\end{array}$ & $\begin{array}{l}264 \\
240 \\
246 \\
360\end{array}$ & $\begin{array}{l}200 \\
200 \\
206 \\
300\end{array}$ & $\begin{array}{l}\text { MOCN } \\
\text { WLCXL } \\
\text { SPRT } \\
\text { EUTW }\end{array}$ \\
\hline $\begin{array}{l}29 \\
30 \\
31 \\
32\end{array}$ & $\begin{array}{l}\text { LEE } \\
\text { LEFLORE } \\
\text { LOWNDES } \\
\text { MADISON }\end{array}$ & $\begin{array}{l}0115 \\
\text { L154 } \\
\text { B030 } \\
\text { U028 }\end{array}$ & $\begin{array}{l}\text { CASON W A } \\
\text { GREENWOOD } \\
\text { CALEDONIA } \\
\text { DEPT OF WILDLIFE }\end{array}$ & $\begin{array}{l}34^{\circ} 0624^{\prime \prime} \\
33^{\circ} 3050^{\prime \prime} \\
33^{\circ} 41^{\prime} 0^{\prime \prime} \\
32^{\circ} 3350^{\prime \prime}\end{array}$ & $\begin{array}{l}88^{\circ} 3649^{\prime \prime} \\
90^{\circ} 11^{\prime} 00^{\prime \prime} \\
88^{\circ} 19^{\prime} 30^{\prime \prime} \\
89^{\circ} 55^{\prime} 10^{\prime \prime}\end{array}$ & $\begin{array}{l}300 \\
200 \\
323 \\
211\end{array}$ & $\begin{array}{l}250 \\
160 \\
283 \\
171\end{array}$ & $\begin{array}{l}\text { MaYN' } \\
\text { SPRT } \\
\text { GORD } \\
\text { COKF }\end{array}$ \\
\hline $\begin{array}{l}33 \\
34 \\
35 \\
36\end{array}$ & $\begin{array}{l}\text { MARION } \\
\text { MARSHALL } \\
\text { RANKIN } \\
\text { SMIIH }\end{array}$ & $\begin{array}{l}\text { L002 } \\
\text { P065 } \\
\text { F067 } \\
\text { P013 }\end{array}$ & $\begin{array}{l}\text { COUUMBIA } \\
\text { LAKE CENTER } \\
\text { REFLECTION APTS } \\
\text { OKATOMA W A }\end{array}$ & $\begin{array}{l}31^{\circ} 14^{\prime} 48^{\prime \prime} \\
34^{\circ} 41^{\prime} 20^{\prime \prime} \\
32^{\circ} 19^{\prime} 36^{\prime \prime} \\
31^{\prime} 4746^{\prime \prime}\end{array}$ & $\begin{array}{l}89^{\circ} 48^{\prime} 50^{\prime \prime} \\
89^{\prime} 21^{\circ} 59^{\prime \prime} \\
9^{\circ} 06^{\prime} 33^{\prime \prime} \\
89^{\circ} 34^{\prime} 26^{\prime \prime}\end{array}$ & $\begin{array}{l}140 \\
113 \\
350 \\
241\end{array}$ & $\begin{array}{r}110 \\
83 \\
330 \\
201\end{array}$ & $\begin{array}{l}\text { MOCN } \\
\text { MuWX } \\
\text { COKF } \\
\text { CIHIL }\end{array}$ \\
\hline $\begin{array}{l}37 \\
38 \\
39 \\
40\end{array}$ & $\begin{array}{l}\text { TATE } \\
\text { TIPPAH } \\
\text { TISHOMINGO } \\
\text { WARREN }\end{array}$ & $\begin{array}{l}\text { F048 } \\
\text { DO14 } \\
\text { DO52 } \\
\text { E023 }\end{array}$ & $\begin{array}{l}\text { STRAYHORN W A } \\
\text { TIPPLERSVILE W A } \\
\text { BURNSVILLE } \\
\text { VCKSBURG }\end{array}$ & $\begin{array}{l}34^{\prime} 3606^{\prime \prime} \\
34^{\prime} 5359^{*} \\
34^{\prime} 5033^{\prime \prime} \\
324^{\prime} 231^{\prime \prime}\end{array}$ & $\begin{array}{l}90^{\circ} 04^{\prime} 33^{\prime \prime} \\
88^{\circ} 54^{\prime} 37^{\prime \prime} \\
88^{\circ} 19^{\prime \prime 22^{\prime \prime}} \\
9^{\circ} 53^{\prime} 02^{\prime \prime}\end{array}$ & $\begin{array}{l}316 \\
190 \\
200 \\
122\end{array}$ & $\begin{array}{r}276 \\
130 \\
220 \\
82\end{array}$ & $\begin{array}{l}\text { SPRT } \\
\text { RPLY } \\
\text { PLZC } \\
\text { MRVA }\end{array}$ \\
\hline $\begin{array}{l}41 \\
42 \\
43\end{array}$ & $\begin{array}{l}\text { WAYNE } \\
\text { WILKNSON } \\
\text { WINSTON }\end{array}$ & $\begin{array}{l}\text { N1S5 } \\
\text { OO23 } \\
\text { FOO1 }\end{array}$ & $\begin{array}{l}\text { WAYNESBORO } \\
\text { CENIREVILLE W A } \\
\text { BOND W A }\end{array}$ & $\begin{array}{l}31^{\circ} 39^{\circ} 55^{\prime \prime} \\
31^{\circ} 05^{\prime} 14^{\prime \prime} \\
33^{\circ} 0744^{\prime \prime}\end{array}$ & $\begin{array}{l}88^{\circ} 38^{\circ} 45^{\prime \prime} \\
91^{\circ} 03^{\prime} 57^{\prime \prime} \\
88^{\circ} 5738^{\prime \prime}\end{array}$ & $\begin{array}{l}138 \\
208 \\
206\end{array}$ & $\begin{array}{r}88 \\
168 \\
181\end{array}$ & $\begin{array}{l}\text { WSBR } \\
\text { CRNL } \\
\text { WLCXI }\end{array}$ \\
\hline
\end{tabular}


Table 3. Values for tritium and selected water-quality properties and constituents for ground water

IpCi/L, picocuries per liter; $\mathrm{TU}$, tritium units; $\mu \mathrm{S} / \mathrm{cm}$, microsiemens per centimeter at 25 degrees Celsius; mg/L, milligrams per liter; $\mathrm{CaCO}_{3}$, calcium carbonate; <, less than]

\begin{tabular}{|c|c|c|c|c|c|c|}
\hline \multirow[t]{2}{*}{$\begin{array}{c}\text { Site } \\
\text { number }\end{array}$} & \multirow[t]{2}{*}{ Date } & \multicolumn{2}{|c|}{$\begin{array}{c}\text { Tritium } \\
\text { concentration }\end{array}$} & \multirow{2}{*}{$\begin{array}{c}\text { Specific } \\
\text { onductance } \\
(\mu S / c m)\end{array}$} & \multirow[t]{2}{*}{$\frac{\mathrm{pH}}{\text { (units) }}$} & \multirow{2}{*}{$\begin{array}{c}\text { Alkalinity } \\
\text { (mg/L as } \\
\mathrm{CaCO}_{3} \text { ) }\end{array}$} \\
\hline & & $(\mathrm{pCi} / \mathrm{L})$ & (TU) & & & \\
\hline $\begin{array}{l}1 \\
2 \\
3 \\
4 \\
5\end{array}$ & $\begin{array}{l}04-20-89 \\
04-20-90 \\
05-03-90 \\
04-18-89 \\
04-20-89\end{array}$ & $\begin{array}{c}41 \\
<.3 \\
<.3 \\
20 \\
4.3\end{array}$ & $\begin{array}{l}13 \\
<.1 \\
<.1 \\
6.2 \\
1.3\end{array}$ & $\begin{array}{r}558 \\
80 \\
340 \\
117 \\
486\end{array}$ & $\begin{array}{l}7.4 \\
6.5 \\
8.5 \\
5.5 \\
7.0\end{array}$ & $\begin{array}{r}288 \\
28 \\
168 \\
8 \\
246\end{array}$ \\
\hline $\begin{array}{r}6 \\
7 \\
8 \\
9 \\
10\end{array}$ & $\begin{array}{l}04-25-90 \\
04-26-89 \\
04-19-89 \\
04-19-89 \\
04-23-90\end{array}$ & $\begin{array}{c}<.3 \\
<.3 \\
34 \\
38 \\
2.0\end{array}$ & $\begin{array}{c}<.1 \\
<.1 \\
11 \\
12 \\
.6\end{array}$ & $\begin{array}{r}450 \\
465 \\
61 \\
40 \\
66\end{array}$ & $\begin{array}{l}8.5 \\
7.2 \\
5.6 \\
5.6 \\
7.2\end{array}$ & $\begin{array}{r}242 \\
251 \\
7 \\
6 \\
25\end{array}$ \\
\hline $\begin{array}{l}11 \\
12 \\
13 \\
14 \\
15\end{array}$ & $\begin{array}{l}04-23-90 \\
04-17-90 \\
04-23-90 \\
04-25-89 \\
04-26-90\end{array}$ & $\begin{array}{l}6.8 \\
<.3 \\
<.3 \\
<.3 \\
<.3 \\
<.3\end{array}$ & $\begin{array}{l}2.1 \\
<.1 \\
<.1 \\
<.1 \\
<.1\end{array}$ & $\begin{array}{r}67 \\
354 \\
354 \\
338 \\
537\end{array}$ & $\begin{array}{l}7.5 \\
8.6 \\
7.6 \\
7.5 \\
8.5\end{array}$ & $\begin{array}{r}19 \\
163 \\
161 \\
148 \\
228\end{array}$ \\
\hline $\begin{array}{l}16 \\
17 \\
18 \\
19 \\
20\end{array}$ & $\begin{array}{l}04-20-89 \\
04-19-89 \\
04-18-89 \\
04-25-90 \\
04-16-90\end{array}$ & $\begin{array}{l}<.3 \\
<.3 \\
<.3 \\
<.3 \\
<.3 \\
<.3\end{array}$ & $\begin{array}{l}<.1 \\
<.1 \\
<.1 \\
<.1 \\
<.1\end{array}$ & $\begin{array}{r}581 \\
65 \\
938 \\
678 \\
154\end{array}$ & $\begin{array}{l}7.2 \\
6.2 \\
7.9 \\
8.3 \\
7.1\end{array}$ & $\begin{array}{r}179 \\
24 \\
299 \\
263 \\
65\end{array}$ \\
\hline $\begin{array}{l}21 \\
22 \\
23 \\
24 \\
25\end{array}$ & $\begin{array}{l}04-21-89 \\
04-25-89 \\
04-19-89 \\
04-19-89 \\
04-19-89\end{array}$ & $\begin{array}{l}31 \\
42 \\
14 \\
31 \\
<.3\end{array}$ & $\begin{array}{c}9.7 \\
13 \\
4.4 \\
9.7 \\
<.1\end{array}$ & $\begin{array}{l}36 \\
85 \\
23 \\
20 \\
30\end{array}$ & $\begin{array}{l}5.3 \\
5.7 \\
5.7 \\
5.5 \\
6.2\end{array}$ & $\begin{array}{r}2 \\
13 \\
5 \\
3 \\
11\end{array}$ \\
\hline $\begin{array}{l}26 \\
27 \\
28 \\
29 \\
30\end{array}$ & $\begin{array}{l}04-25-90 \\
04-20-90 \\
04-24-90 \\
04-24-90 \\
04-25-89\end{array}$ & $\begin{array}{r}<.3 \\
<.3 \\
.6 \\
<.3 \\
.9\end{array}$ & $\begin{array}{r}<.1 \\
<.1 \\
2 \\
<.1 \\
.3\end{array}$ & $\begin{array}{r}245 \\
80 \\
390 \\
335 \\
455\end{array}$ & $\begin{array}{l}8.0 \\
6.5 \\
8.2 \\
8.0 \\
7.2\end{array}$ & $\begin{array}{r}113 \\
14 \\
108 \\
158 \\
234\end{array}$ \\
\hline $\begin{array}{l}31 \\
32 \\
33 \\
34 \\
35\end{array}$ & $\begin{array}{l}04-20-89 \\
04-26-90 \\
04-19-89 \\
04-24-90 \\
04-19-90\end{array}$ & $\begin{array}{l}<.3 \\
13 \\
12 \\
30 \\
<.3\end{array}$ & $\begin{array}{l}<.1 \\
4.1 \\
3.8 \\
9.4 \\
<.1\end{array}$ & $\begin{array}{r}65 \\
145 \\
43 \\
37 \\
507\end{array}$ & $\begin{array}{l}6.2 \\
7.0 \\
5.8 \\
6.7 \\
8.4\end{array}$ & $\begin{array}{r}25 \\
36 \\
9 \\
10 \\
211\end{array}$ \\
\hline $\begin{array}{l}36 \\
37 \\
38 \\
39 \\
40\end{array}$ & $\begin{array}{l}04-16-90 \\
04-25-89 \\
04-18-89 \\
04-19-89 \\
04-20-89\end{array}$ & $\begin{array}{l}28 \\
<.3 \\
<.3 \\
<.3 \\
17\end{array}$ & $\begin{array}{l}8.8 \\
<.1 \\
<.1 \\
<.1 \\
5.3\end{array}$ & $\begin{array}{r}53 \\
91 \\
370 \\
61 \\
503\end{array}$ & $\begin{array}{l}5.9 \\
6.3 \\
7.8 \\
6.2 \\
7.1\end{array}$ & $\begin{array}{r}4 \\
38 \\
192 \\
20 \\
258\end{array}$ \\
\hline $\begin{array}{l}41 \\
42 \\
43\end{array}$ & $\begin{array}{l}05-03-90 \\
05-04-90 \\
04-25-90\end{array}$ & $\begin{array}{l}<.3 \\
<.3\end{array}$ & $\begin{array}{l}<.1 \\
6.6 \\
<.1\end{array}$ & $\begin{array}{r}327 \\
99 \\
33\end{array}$ & $\begin{array}{l}8.3 \\
6.4 \\
6.6\end{array}$ & $\begin{array}{r}159 \\
14 \\
7\end{array}$ \\
\hline
\end{tabular}


Table 4. Median values of depth, specific conductance, $\mathrm{pH}$, and alkalinity for pre- and post-1953 ground water [based on 1989-90 tritium data]

\begin{tabular}{lcccc}
\hline & \multicolumn{4}{c}{ Median values } \\
\cline { 2 - 5 } & $\begin{array}{c}\text { Depth } \\
\text { (feet below } \\
\text { land surface) }\end{array}$ & $\begin{array}{c}\text { Specific conductance } \\
\text { (microsiemens per } \\
\text { centimeter at 25 } \\
\text { degrees Celsius) }\end{array}$ & $\begin{array}{c}\text { pH } \\
\text { (standard } \\
\text { units) }\end{array}$ & $\begin{array}{c}\text { Alkalinity } \\
\text { (milligrams } \\
\text { per liter as } \\
\text { calcium carbonate) }\end{array}$ \\
\hline Post-1953 water & 187 & 66 & 5.9 & 10 \\
Pre-1953 water & 277 & 339 & 7.6 & 158 \\
\hline
\end{tabular}

